\section{GWHR Conference Abstracts}

\section{Global Trends in Women's Health: Challenges and Opportunities}

\author{
Monique Chireau, M.D., M.P.H. \\ Assistant Professor of Obstetrics \\ and Gynecology \\ Duke University School \\ of Medicine
}

After a period of restrictions on international abortion funding by the U.S. government, following the 2008 election, a new emphasis has been placed on promoting abortion worldwide. Five major contributors to abortion globalization have been identified: 1) policy of developed nations to expand abortion in developing nations through linkage of funding to women's health agendas promoting abortion; 2) the use of research activism and legislative activism to pressure for abortion legalization; 3) rapid shifts in political power worldwide with resultant instability in cultural mores, leaving nations vulnerable to population control ideologies; 4) imprecise and quasi-legal definitions of critically important terms, such as "abortion," "unsafe abortion," "spontaneous abortion," and "induced abortion," allowing for ambiguous and contradictory conclusions in research and statistics; and 5) rapid technological changes such as increased portability of MVA and introduction of mifepristone, misoprostol, and ulipristal.

There are a variety of problems with the globalization plan. Most data supporting abortion as a significant cause of maternal mortality are deeply flawed. Terminology used for definitions and outcomes of abortion is misleading or inaccurate. Data are skewed and manipulated in such a way as to misrepresent statistical methods, data sources and conclusions that can reasonably be drawn from the data. Ultimately policy, funding decisions and public perceptions are affected by these statistics. There is, therefore, a need for accurate data. It is important for the scientific community to refute inaccurate data or inappropriate use of statistics and to publish high-quality research, since research has an impact on public policy. There is also a need for standardized nomenclature and accurate statistics on the local level to support programmatic planning. The ultimate goal is to improve women's health. Therefore, the scientific community needs to establish standard nomenclatures for abortion and its subtypes. It must also develop a system for standardized collection of data as part of vital statistics. Finally, research on incidence and prevalence of maternal mortality and morbidity (MMM) must continue in order to assess the efficacy of interventions to reduce MMM without promoting induced abortion.

\section{Current Models for Research in Global Women's Health in Peru}

\author{
Paul Ramos, M.D. \\ Peru
}

An analysis of maternal mortality statistics from 1955 until 2000 demonstrates a steady decline in maternal mortality since the inception of maternal mortality recordkeeping. A closer analysis of the number of annual maternal deaths from 1997 to 2008 reveals a decline from 769 to 505. Region-specific analysis of maternal deaths reveals 80 percent of maternal

The Linacre Quarterly 78(2) (May 2011): 192-194.

(C) 2011 by the Catholic Medical Association. All rights reserved. 0024-3639/2011/7802-0008 \$.30/page. 
deaths are in regions without easy access to skilled birth attendance, and maternal mortality is significantly lower in regions with fewer barriers to skilled birth attendance. Detailed analysis of causality reveals approximately 40 percent of deaths are due to peripartum hemorrhage, and only 6 percent due to abortion, either spontaneous or induced. Proponents of legalization of abortion attribute a majority of maternal mortality to abortion in Peru, but without any objective justification for this attribution. Rather, an evidence-based analysis reveals that maternal mortality in Peru has an inverse correlation to the availability of skilled birth attendance. The road to death for Peruvian women is one with roadblocks to access. To decrease maternal mortality in Peru, greater resources need to be spent in providing access to skilled birth attendance, antibiotics, and oxytocic drugs to the peripartum woman. Importing a European model of abortion legalization does not fit the reality of maternal mortality in Peru.

\section{Is There an Association Between Maternal Mortality Reduction and Abortion Legalization? Preliminary Data from Time Series Analyses in Chile, 1960-2007}

Elard Koch, M.Sc., Ph.D. Department of Family Medicine, Faculty of Medicine University of Chile Santiago, Chile

and

Jorge Reyes, Esq., J.D. Chile

Background: Nowadays, the impact of abortion prohibition on maternal mortality trends in developing countries is unknown. Chile offers the opportunity for assessing the impact of different factors related with maternal mortality reduction, including two periods before and after abortion was prohibited in this Latin American country.
Methods: Time series of maternal mortality ratio (MMR) and abortion mortality ratio (AMR) from 1960 to 2007 were analyzed using multiple autoregressive moving average (ARIMA) models. Therapeutic abortion was legal until 1989 and was considered as a dummy variable in statistical analyses along time series of social and demographic factors and maternal health facilities.

Results: During the study period, MMR decreased from 293.7 to 18.2 per 100,000 live births (-93.8\%); AMR decreased from 92.5 to 1.7 per 100,000 live births $(-98.1 \%)$. No significant effect of legal and illegal abortion periods on these decreasing trends was observed in ARIMA models. After abortion was fully prohibited, MMR and AMR decreased from 41.3 to $18.2(-44.1 \%)$ and 16.5 to 1.7 $(-10.3 \%)$ per 100,000 live births respectively. The average of education years, illiteracy rate, GDP per-capita, and the percentage of delivery by skilled attendants were all significant predictors of MMR. The same factors along decreasing fertility rate were significant predictors of AMR trends.

Conclusions: Reduction in MMR and AMR are not related with legal/illegal therapeutic abortion periods in Chile. The increasing education level appears as the most important factor predicting maternal mortality reduction in this developing country, likely influencing other factors such as fertility and maternal health facilities.

\section{Resolving the Post-Abortion Health Issues Using Available Statistical Data}

\author{
Patrick S. Carroll, M.A. \\ Director of Research \\ Pension and Population \\ Research Institute \\ London, UK \\ papriresearch@btconnect.com
}

The myth of "safe" abortion continues despite a large and growing body of 
research linking induced abortion to preterm birth, breast cancer, depressive disorders, and other sequelae. After forty years of induced abortion, some comprehensive data sets exist in several countries. European data from several countries including England, Wales, Scotland, and the Scandinavian nations is more comprehensive than that available in the United States, and represent a complete account of legal induced abortions along with age and other demographic details. To investigate effectively the medical and social impact of abortion on a population, the demographic pattern and profile of modern abortion provides useful information in situations where the methods of Bradford Hill are difficult to apply. Demographic grouping of women who have increased vs. lower rates of abortion, such as Black American women with a threefold higher abortion rate than Caucasian American women, allows for inferences from correlation analysis in ecological studies. Consideration of the sequence of abortion in parity history allows detection of specific disorders such as breast cancer, which is linked most strongly to termination of nulliparous pregnancies. Although a large amount of research has established the linkage between induced abortion and subsequent preterm birth, potential exists for more precise delineation of the interaction between known risk factors.

\section{Interfacing and Collaboration of Pro-Life Legal Efforts}

\section{Presentation by William L. Saunders at AAPLOG strategy meeting on January 14, 2009}

William Saunders, senior vice-president and senior counsel at Americans United for Life, outlines a collaborative legal approach for the pro-life movement to counter the strategy that has been used by the Left to promote abortion as a "right" in international law, binding upon each and every sovereign nation.

Saunders explains what is meant by international law and cites several recent examples from around the globe of the abortion lobby's attempt to distort it to include a right to abortion.

Saunders suggests that to stop the misuse of international law against the unborn, the pro-life movement must work internationally and collaboratively. He describes the international project launched by Americans United for Life to help pro-life forces in other nations resist the "international law assault" and help develop pro-life laws in other countries.

Saunders concludes that pro-lifers can, and must, be active everywhere, making the pro-life case in all its dimensions, ensuring that the pro-life message is heard. 